\title{
Frequency and Temporal Identification of a Li-ion Polymer Battery Model Using Fractional Impedance
}

\author{
M. Montaru* and S. Pelissier \\ INRETS - Institut National de la Recherche sur les Transports et la Sécurité, 25 avenue F. Mitterrand, Case 24, 69675 Bron Cedex - France \\ e-mail: maxime.montaru@inrets.fr - serge.pelissier@inrets.fr \\ * Corresponding author
}

Résumé - Identification fréquentielle et temporelle d'un modèle de batterie Li-polymère utilisant une impédance fractionnaire - La modélisation des batteries lithium de fortes puissances est un moyen performant de dimensionner le pack de batterie des véhicules hybrides. De plus, cet outil peut être utilisé pour évaluer précisément les caractéristiques d'une batterie à chaque étape de sa vie. Jusqu'à maintenant, les structures de modèles diffèrent selon l'utilisation du modèle et les limitations de la simulation. Il apparaît au travers des mesures de spectroscopie d'impédance que le modèle doit comprendre une impédance d'ordre non entier. Or, ce type d'impédance ne peut pas être simulé par les logiciels dédiés aux véhicules hybrides et nécessite donc une approximation. Par ailleurs, les mesures d'impédance sont réalisées à faibles courants par rapport aux intensités observées en usage normal, ce qui peut induire des erreurs de modélisation dans le cas où l'impédance varie avec le courant. Ce papier présente un modèle qui utilise une impédance d'ordre non entier de gamme de fréquence bornée et qui prend en compte le phénomène de non linéarité. Les paramètres sont identifiés partiellement par spectroscopie d'impédance pour la partie haute fréquence et par chronopotentiométrie pour la partie basse fréquence dans une large gamme d'intensité. Le modèle est validé avec des échelons de courant de fortes intensités et un profil de courant simulé sur un logiciel de simulation de véhicule hybride. L'hypothèse de non linéarité est vérifiée.

\begin{abstract}
Frequency and Temporal Identification of a Li-ion Polymer Battery Model Using Fractional Impedance - The modelling of high power Lithium batteries may work as a powerful tool in the sizing of batteries pack in hybrid vehicles. Moreover, this tool can be used to evaluate accurately the battery characteristics at each level of its lifetime. Until now, the model structure differs according to its end-use due to simulation limitation. Indeed, impedance measurements obtained by electrochemical impedance spectroscopy needs non-integer order impedance in order to correctly model diffusive phenomenon. However, this impedance cannot be simulated easily in software dedicated to hybrid vehicles and needs approximations. Furthermore, impedance measurements are realised with low currents comparing to currents that are forecast in normal uses of high power batteries and can induce errors if the impedance is varying with the current. This paper deals with non linear dynamic models that use band-limited frequency impedance with non-integer order. The parameters are identified partly by impedance measurements for high frequencies and partly by chronopotentiometry measurements for low frequencies. Validation tests are made with staircase of high intensity and current profile simulated on a hybrid vehicle software. The hypothesis of non-linearity is verified.
\end{abstract}




\section{INTRODUCTION}

The battery is one of the essential parts in a Hybrid Electric Vehicle (HEV) and probably the most critical one with respect to reliability and life expectation. It is a primordial element in the concept of hybridation because it enables to reversibly store energy. The energy stored is picked up during deceleration of the vehicle and used for starting the thermal engine and giving a boost during acceleration. The battery can be used in a sustaining mode, i.e. in a small window of State Of Charge (SOC), or in a depleting mode, i.e. in a large window of SOC. The second case enables a succession of high power discharges which permits the vehicle to reduce much more its consumption or even to stop completely the thermal engine. The power supplied and received by the battery is variable and could be very important depending notably on the sizing of the pack of batteries and its management. In order to optimize its sizing and to maintain a correct lifetime, the knowledge of the model of the electric storage components and their dynamic response is necessary.

The modelling of batteries is not an easy task. The reasons are [1]:

- batteries are not stationary due to the SOC variation and diffusion phenomenon;

- they are systems highly non-linear regarding to the current, temperature, etc.

Two families of identification of the model parameters, temporal and frequency, are currently used but not necessarily for the same purpose.

In one hand, it is possible to use the temporal chronopotentiometry. This technique consists of applying a pulse of current and measuring the voltage of the elements. It's the method used by the Patnership of the New Generation of Vehicle [2] who names it High Power Pulse Characterization. The aim is to evaluate the resistance of batteries after a certain time of applied current. It can also be used to identify much more complex model as it was proposed in the study with ADVISOR models [3]. It's also possible to use profile of current to model dynamic behaviour. This profile can be a pseudo random binary sequence [4] or simply represent a profile of current which could be measured on a HEV [5]. In the other hand, a well-known technique for impedance characterization is impedance spectroscopy [6]. This technique can be used to identify relationship between material properties and battery performance if just one interface is studied [7, 8]. It is risky to do the same with a complete cell because it's not possible to dissociate the effect of the cathode from the one of the anode. However, it is possible to associate the impedance measured by spectroscopy to the state of charge, or even state of health $[9,10]$. Moreover, the impedance of a battery cell has been identified with this technique [11]. Currently, in electrochemical studies, the voltage oscillation is imposed around the Open Circuit Voltage $(O C V)$ but in case of commercial cell, it is possible to impose current oscillation and to control state of charge during the operations. The measurement of impedance can be done with or without current polarization. When the spectrometer can supply sufficient continuous current, it is possible to highlight non linearity of the battery impedance with the current level $[1,9,10,12,13,14]$. Another frequency method to identify battery impedance consists of using Laplace transform of temporal solicitations $[15,16]$.

The advantage of chrono-potentiometry is to allow discharge and charge of high current level as in normal use. However, the acquisition frequency may be limited by the equipment and prevents from identifying correctly the impedance at high frequencies. On the contrary, impedance spectroscopy gives a precise impedance measurement in a wide band of frequencies but only for low current values. In order to conjugate the advantage of each technique, the method used in this study consists of a partial identification of the model by spectroscopy impedance and the rest by chronopotentiometry. It enables a gain of time due to the less time spent in impedance spectroscopy at low frequencies and a more complete model with the non-linearity of impedance with current. Furthermore the equipment is standard unlike some other works $[13,14]$.

\section{IMPEDANCE-BASED BATTERY MODEL}

\subsection{Full Model for Identification}

The model used in this study is an equivalent electric circuit with lumped parameters. In such a model, parameters could

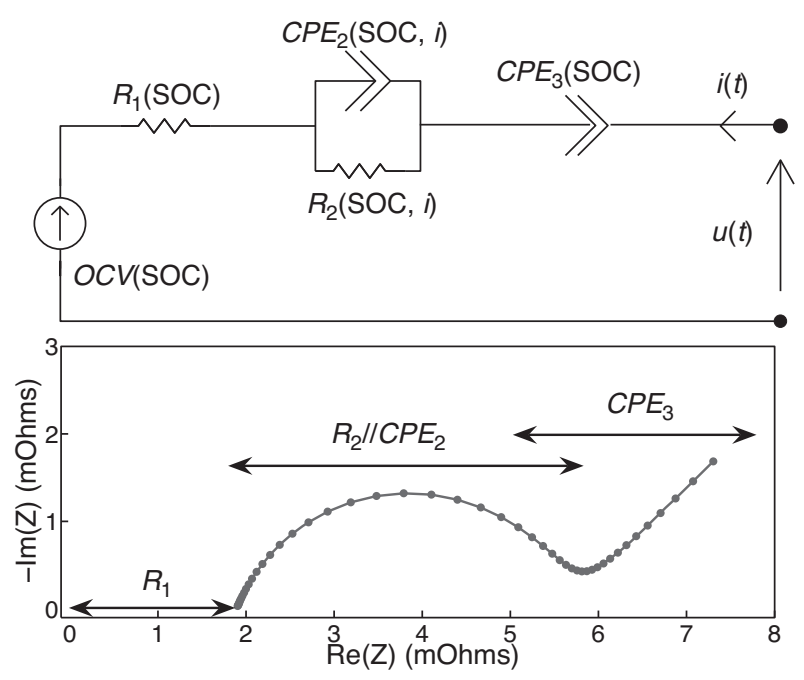

Figure 1

Simplified electric circuit model and its representation in Nyquist plan. 
be tabulated according to SOC, current, temperature, etc. $[13,17]$. A representation of our model depending on SOC and current is given in Figure 1. It consists of an open circuit voltage $O C V$ in series with a complex impedance. The tabulation of the $O C V$ according to $\mathrm{SOC}$ could be a very difficult task if hysteresis phenomenon occurs as observed with NiMH batteries [13], but it is not the case in our study since hysteresis is very small in this type of Li-ion battery. The complex impedance is composed of 3 elements in series: a resistance $R_{1}$ for modelling the ohmic behaviour, a resistance $R_{2}$ in parallel with a Constant Phase Element $C P E_{2}$ for modelling the charge transfer and double layer processes and another Constant Phase Element $\mathrm{CPE}_{3}$ associated to the diffusion behaviour. This impedance model is adapted to fit well with impedance spectrum (cf. Fig. 1). In many studies, the diffusive impedance is represented by a Warburg impedance which expression, in frequency domain, is:

$$
Z(j \omega)=\frac{1}{Q \sqrt{j \omega}} \tanh (\sqrt{j \tau \omega})
$$

$[14,17]$. However, in our model, we used a simplified expression of the Warburg impedance obtained with a constant phase element $C P E_{3}$. The difference between these two expressions occurs at very low frequencies not reached in this study.

\subsection{Modelling the Constant Phase Element $\mathrm{CPE}_{3}$}

A CPE consists of non integer order impedance which is expressed in the frequency domain as:

$$
C P E(j \omega)=\frac{1}{Q \cdot(j \omega)^{\alpha}}
$$

with $\omega$ the pulsation in rad. $\mathrm{s}^{-1}, \alpha$ a real number comprised between 0 and 1 and $Q$ a constant expressed in $F . s^{\alpha-1}$. In the case of $\alpha=1, C P E$ is equivalent to a capacitance. In the case of $\alpha=0, C P E$ is equivalent to a resistance. A $C P E$ is represented as a straight line in Nyquist plan (Fig. 1) and in Bode diagram (Fig. 2). Simulation in temporal mode necessitates finding an equivalent circuit of $C P E$. Several authors proposed to use a mathematical series of $R / / C$ circuits as an equivalent of the diffusive Warburg impedance [14, 17]. Based on the same principle, a CPE could be approximated by a fractional impedance in a limited frequency band $[4$, 18]. It consists of several $R / / C$ circuits which frequency characteristics are judiciously chosen in order to approximate a straight line only in a limited frequency band $\left[\omega_{\min }, \omega_{\max }\right]$ as shown in Figure 2. In this work, we used five $R / / C$ as a good compromise between accuracy and duration of computation. The expression of the impedance is given by:

$$
Z(j \omega)=\frac{\gamma}{1+\frac{j \omega}{\omega_{1}} \cdot \frac{1+\frac{j \omega}{\omega_{1}^{\prime}}}{1+\frac{j \omega}{\omega_{2}}} \cdot \ldots} \cdot \frac{1+\frac{j \omega}{\omega_{4}^{\prime}}}{1+\frac{j \omega}{\omega_{5}}}
$$

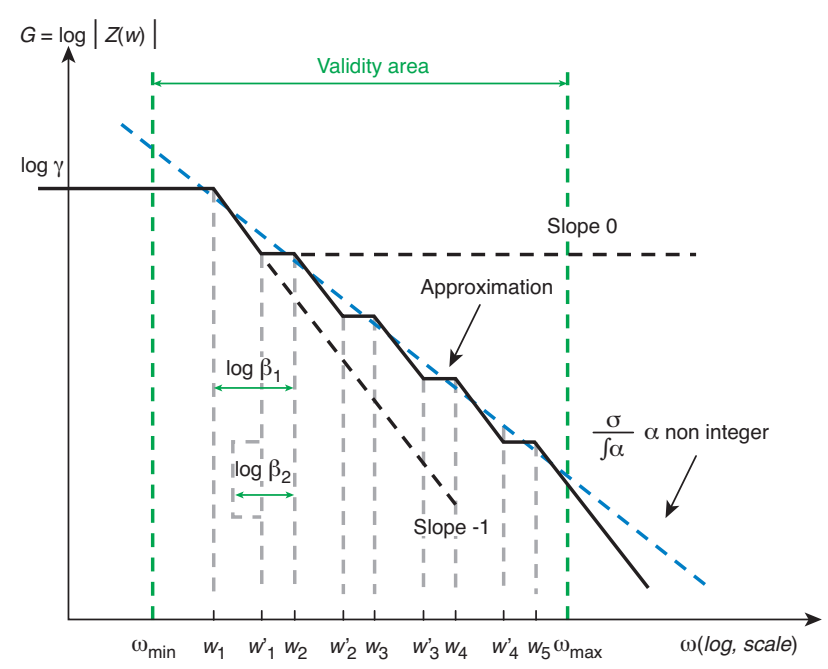

Figure 2

Bode diagram of the approximation of the diffusive impedance by 5 circuits $R / / C$.

The pulsations $\omega_{i}$ and $\omega^{\prime}{ }_{i}$ of each $R / / C$ circuits are depending on the others by these relations:

$$
\begin{aligned}
& \log \beta_{1}=\log \omega_{i+1}-\log \omega_{i}^{\prime} \\
& \log \beta_{2}=\log \omega_{i+1}-\log \omega_{i}^{\prime}
\end{aligned}
$$

The slope of the approximated impedance in Bode diagram is given by:

$$
p=1-\frac{\log \beta_{1}}{\log \beta_{2}}
$$

Then, as one can see on a Bode diagram (Fig. 2), the gain of the approached line is fixed by $\gamma$ when the pulsation tends to zero, i.e. $Z(\omega \rightarrow 0)=\gamma$.

In summary, a $C P E$ which is defined by $Q$ and $\alpha$ is approximated in the frequency band $\left[\omega_{\min }, \omega_{\max }\right]$ by an impedance defined by four parameters $\left(\omega_{1}, \omega_{5}, \gamma, p\right)$.

\subsection{Modelling $R_{2} / / C P E_{2}$}

The impedance of the circuit $R_{2} / / C P E_{2}$, also named ZARC [6], is expressed by:

$$
Z_{2}(j \omega)=\frac{R_{2}}{1+\tau_{2}(j \omega)^{\alpha_{2}}} \text { with } \tau_{2}=R_{2} Q_{2}
$$

It is possible to extract the value of these parameters from an impedance spectrum ( $c f$. Fig. 3). Firstly, the intersection of the impedance spectrum with the abscissa line enables to detect $R_{1}$. Secondly, the spot at top of the semicircle is characterized by its real part, its imaginary part and its pulsation $\omega_{c, 2}$. Their values can be obtained by measurement. The real part is equal to $R_{1}+0.5 \times R_{2}$ and 


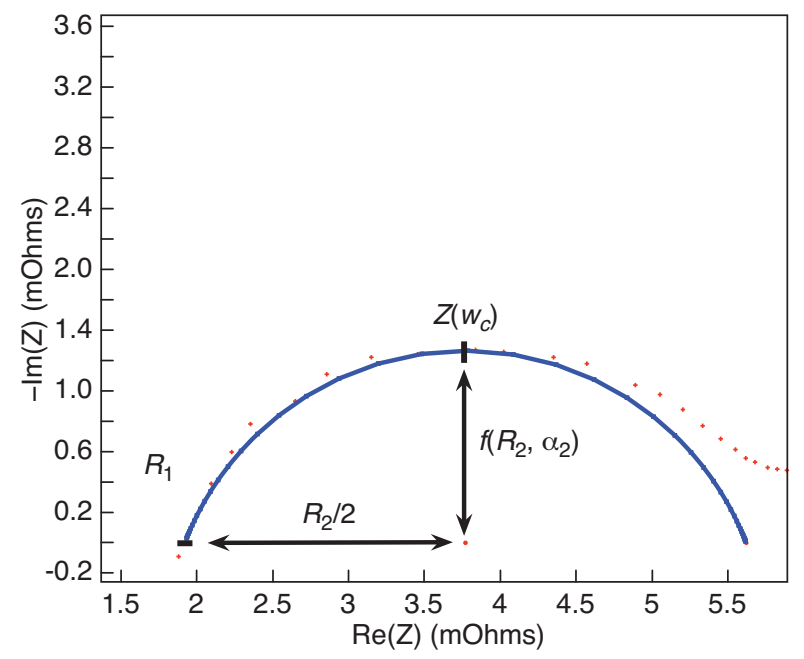

Figure 3

Representation of $Z_{2}$ in the Nyquist plan.

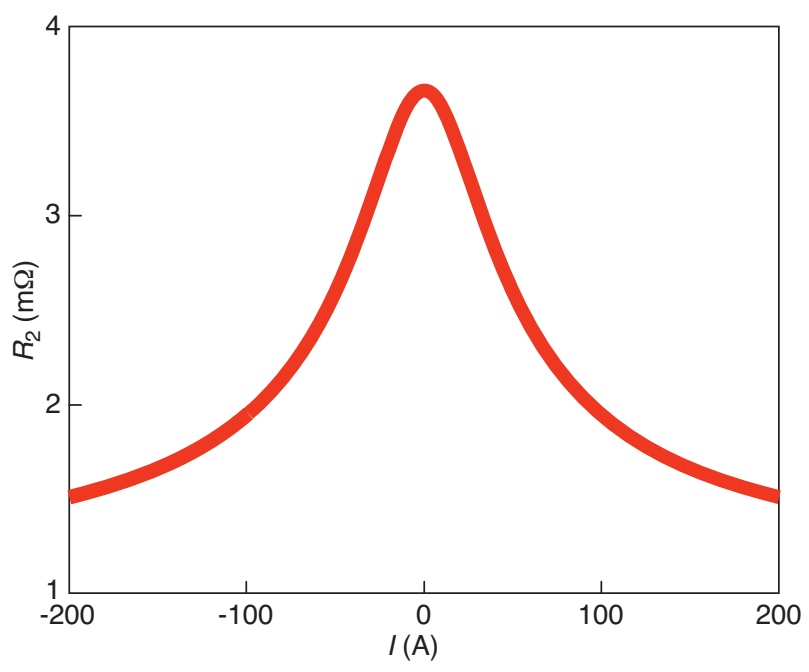

Figure 4

Representation of the fitting function of $R_{2}$. enables to evaluate $R_{2}$. The characteristic pulsation $\omega_{c, 2}$ is linked to the other parameters [6]:

$$
\omega_{c, 2}=\frac{1}{\tau_{2}^{1 / \alpha_{2}}}
$$

$\alpha_{2}$ is obtained from the following expression of the imaginary part of the top of the semi-circle deduced from Equations (6) and (7):

$$
\mathfrak{\Im}_{m}\left(Z_{2}\left(\omega_{c, 2}\right)\right)=R_{2} \frac{\sin \left(\alpha_{2} \cdot \frac{\pi}{2}\right)}{2\left(1+\cos \left(\alpha_{2} \cdot \frac{\pi}{2}\right)\right)}
$$

Then, the parameter $\tau_{2}$ and consequently $Q_{2}$, is deduced from Equation (6) of the pulsation of the top of the semi-circle.

\subsection{Non-linearity of $R_{2}$}

The phenomenon of non-linearity is taken into account for the resistance $R_{2}$. The formalism of Butler-Volmer is considered for this purpose [13]:

$$
i=\frac{A}{2}\left(e^{a B \eta}-e^{-(1-a) B \eta}\right) \text { with } \eta=u-O C V
$$

A hypothesis of symmetry is made concerning the nonlinearity between the charge and the discharge $(a=0.5)$. So:

$$
i=A \sinh (B \eta)
$$

It is possible to deduce an expression of the equivalent resistance:

$$
\frac{1}{R_{2}}=\frac{d i}{d \eta}=A B \cosh (B \eta)
$$

Then, an expression of $R_{2}(i)$ can be derived:

$$
R_{2}(i)=\frac{1}{A B \sqrt{1+\left(\frac{i}{A}\right)^{2}}}+C
$$

For the need of fitting, it appears that it is necessary to add a constant $C$ to $R_{2}$. The representation of this function is given at Figure 4.

\subsection{Simplified Model}

It appears when identifications of these parameters are made that $\tau_{2}$ is comprised between 20 and $30 \mathrm{~ms}$. Considering that this time constant is very small compared to acquisition period of the solicitation profile (100 ms), the model $Z_{2}$ can be simplified. A pure resistance $R_{2}$ replaces the $R_{2} / / C P E_{2}$ which results on a simplified model (cf. Fig. 5) to be used for simulation.

To conclude, at each SOC, the battery model used in temporal simulation is defined by:

- the $O C V$;

- the resistance $R_{1}$;

- the parameters of the non linear model of $R_{2}: A, B, C$;

- the parameters of the diffusive impedance $C P E_{3}: \omega_{1}, \omega_{5}$, $\gamma, p$.

So, 9 parameters are needed to be identified in order to simulate correctly the model. Moreover, the evaluation of each of them could give great information on battery behaviour according to state of charge, temperature and state of health. 


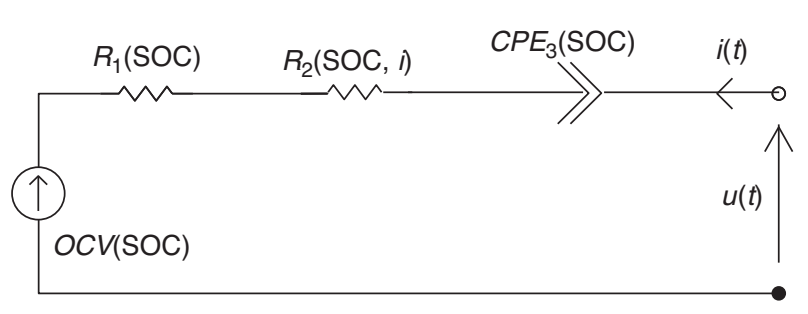

Figure 5

Simplified model of li-ion battery used in temporal simulation.

\section{METHODS \& EXPERIMENTAL SETUP}

The experimental data of this study concern a Li-ion Polymer battery (KOKAM SLPB 702051300P - $12 \mathrm{Ah}$ ) tested at $20^{\circ} \mathrm{C}$.

\subsection{Existing Methods for Identification of the Model of Battery}

As previously said, the two main possibilities to obtain experimental data for identification of the model of a battery are impedance spectroscopy and chronopotentiometry:

- impedance spectroscopy is based on the measurement of complex impedance of the battery at various frequencies $Z(f)=\Re_{e}(f)+i \mathfrak{s}_{m}(f)$. The advantage of this method is the full signature obtained for the battery. Difficulties exist to measure impedance at very low frequencies (below $1 \mathrm{mHz}$ ) because of the duration of the operation, which could last several hours in this case, and the eventual consequently no-stability of the result. Another limitation of this method is the difficulty for measurement of impedance when a DC current is superposed on the AC measurement signal in order to characterize the impedance of the battery during charge or discharge phases. With high values of current, the SOC of the battery could be largely modified during the measurements; in this case the impedance is not constant all along the test and compensation drift procedures must be applied [1, 19];

- chronopotentiometry is based on the voltage response of the battery when a current profile is imposed. The impedance is obtained with the $u(t) / i(t)$ ratio. By using different levels of signals, it could be easy to determine the impedance of the battery for any value of charge or discharge currents. The main limitation of this method is the need for a high sampling rate if one wants to measure the impedance at high frequencies. In practice, two sorts of equipments can be used: either a battery tester with high power capabilities but usually with a limited synchronous sampling period (for example $100 \mathrm{~ms}$ ) or an impedance analyser with high frequencies capability but usually with a limited current (for example $20 \mathrm{~A}$ ). A compromise must be found between frequency and power, with a large incidence on the price of the equipment.

\subsection{Proposed Method for Identification of Power Batteries}

We propose a two step identification process based on two frequency domains. The identification of $R_{1}, R_{2}$ and $C P E_{2}$ is performed at "high frequencies", in the $1 \mathrm{kHz}-500 \mathrm{mHz}$ band, using an impedance analyser. The identification of the elements of the diffusive impedance is obtained by chronopotentiometry with low frequency pulses supplied by a battery test equipment.

\subsubsection{First Step: Characterization of $R_{1}, R_{2}$ and $C P E_{2}$}

The aim is to measure the values of these elements for different values of the SOC of the battery and different values of the charging/discharging current. This current is imposed during a pulse of $20 \mathrm{~s}$. An impedance spectroscopy in the $1 \mathrm{kHz}-500 \mathrm{mHz}$ band with $12 \mathrm{~s}$ duration, is performed $5 \mathrm{~s}$ after the beginning of the pulse. A charging pulse is followed by a discharging pulse to keep SOC constant. This couple of pulse is repeated with several levels of current $(0,6,12$ and $18 \mathrm{~A})$. Measurements are made at different value of SOC $(80,60,40$ and $20 \%)$. The duration of the pulse is shorter enough to consider no variation in the value of SOC $(18 \mathrm{~A} \times 20 \mathrm{~s}=0.1 \mathrm{Ah}$ is less than $1 \%$ of the capacity of the battery). The equipment used for this measurement is a VSP Biologic with a 20 A amplifier kit. An example of the Nyquist graph of impedance for one SOC value and one level of current is shown in Figure 6. The values of $R_{1}, R_{2}, Q_{2}$ and $\alpha_{2}$ are determined with the methods explained in the previous part. As shown in this figure, one needs to interpolate the point which intercepts the abscissa and the one which has the maximum imaginary part. Figure 7 gives the spectroscopy results for different levels of current. One can see that $R_{1}$ is constant and that $R_{2}$ decreases when the current increases. These experimental data are used to identify the parameters of the theoretical relation $R_{2}(i)$ ( $c f . E q .12$ ). As the maximum value of the experimental current is $18 \mathrm{~A}$, it is necessary to obtained at least one data point for higher current in order to have better fitting. This could be achieved by a complementary measurement of chronopotentiometric response to a high intensity pulse. We used a battery tester composed of a DC source (Delta SM30200 - $30 \mathrm{~V}-200 \mathrm{~A}$ ) associated with an electronic load ( $H \& H$ ZS4206 - 60 V - 450 A) to generate short pulses with an amplitude between $50 \mathrm{~A}$ and $200 \mathrm{~A}$ in discharge and between $50 \mathrm{~A}$ and $100 \mathrm{~A}$ in charge. The charging pulse duration is $2 \mathrm{~s}$. One must calculate the duration of the discharging pulse to balance the SOC. An example of the battery voltage during pulse is given by Figure 8 . 


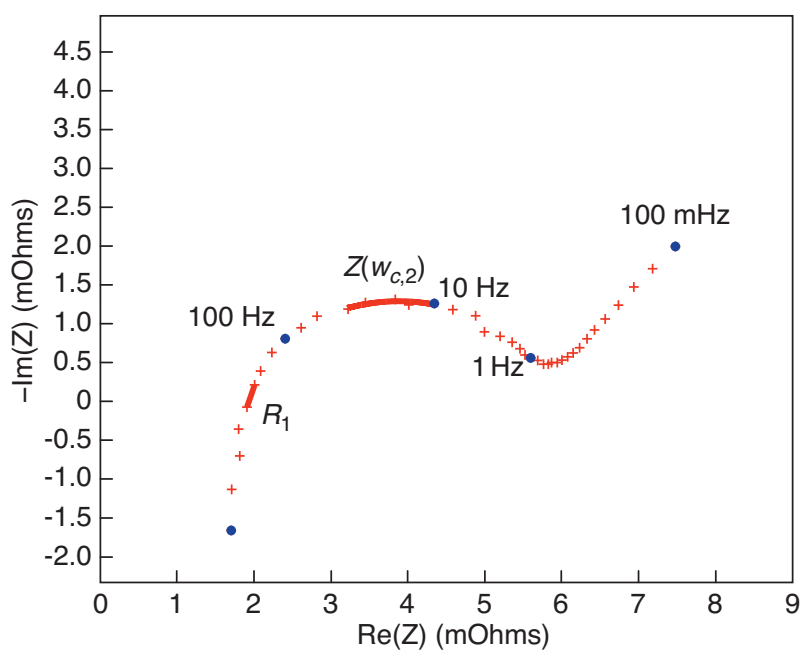

Figure 6

Techniques for identification of $R_{1}$ and $R_{2} / / C P E_{2}$.

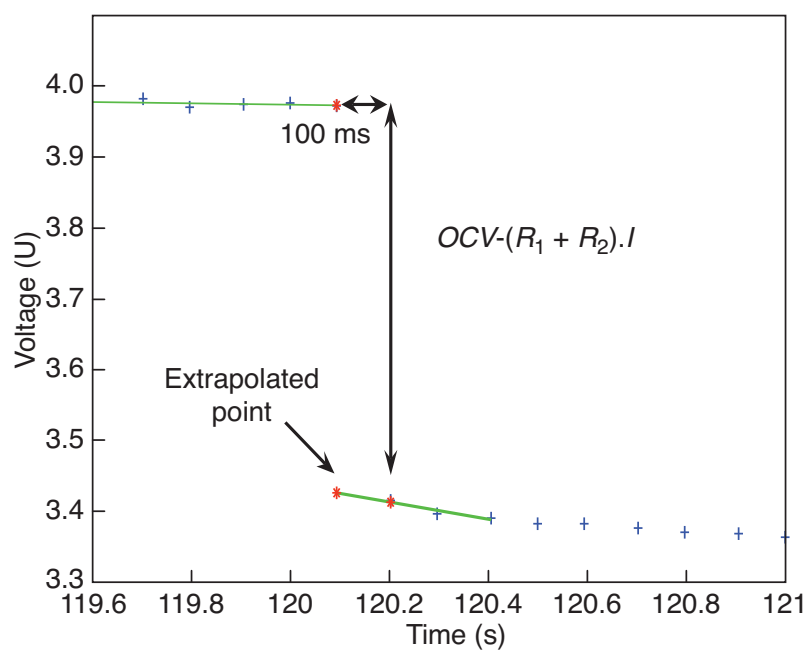

Figure 8

Measured voltage of a Lithium battery on the battery tester during a discharge at $150 \mathrm{~A}$ for SOC of $80 \%$.

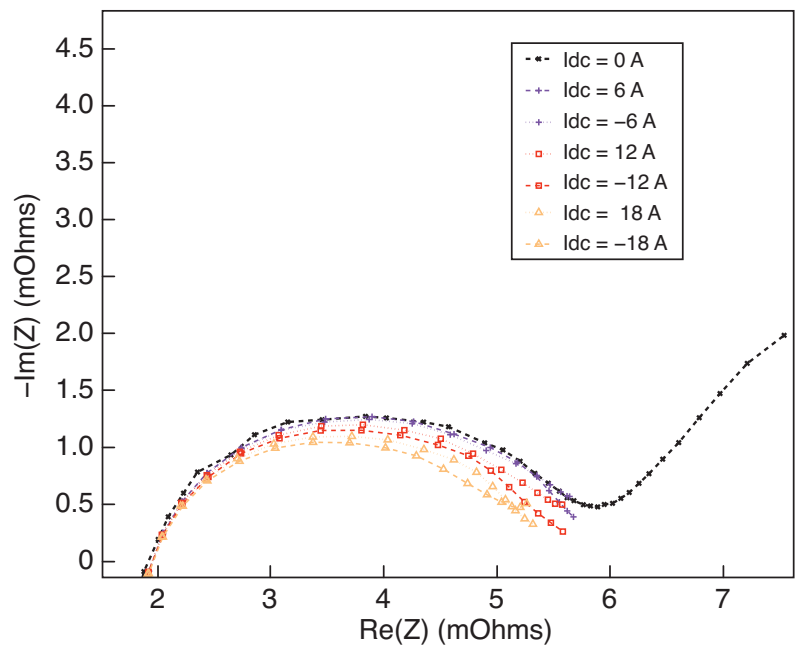

Figure 7

Impedance spectra measured on a Lithium battery for different polarization current levels at SOC $=80 \%$.

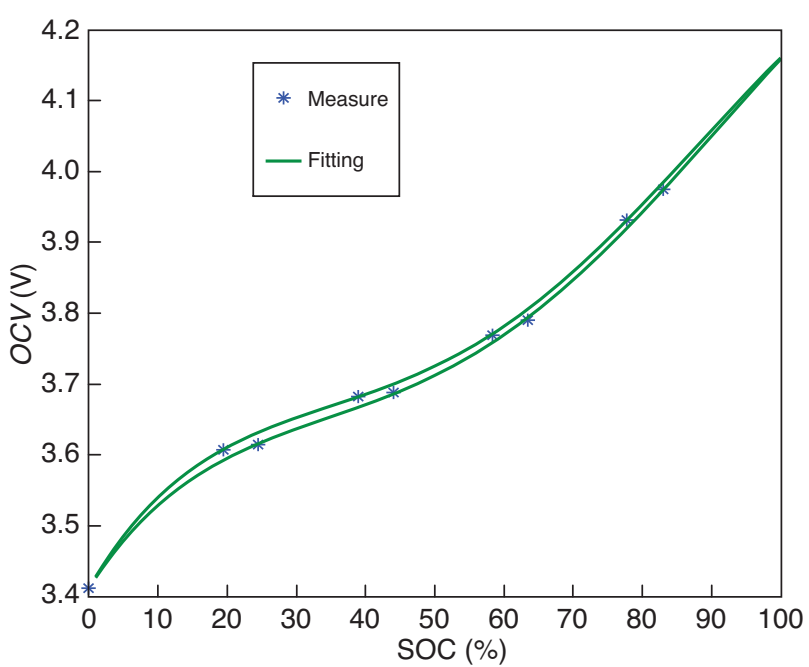

Figure 9

Measured open circuit voltage with verification of hysteresis.
We propose to estimate the value of the resistance from the voltage drop. The sampling frequency $(10 \mathrm{~Hz})$ is not high enough to have numerous data points during the front voltage and a linear extrapolation must be done.

\subsubsection{Second Step: Characterization of the Parameters of the $C P E_{3}$ by Chronopotentiometry}

The aim is to measure the values of these parameters for different SOC and with pulse profile capable of giving information in the $1 \mathrm{~Hz}-1 \mathrm{mHz}$ band. We have chosen to use two 5 min $-1 \mathrm{C}$ charging and discharging pulses preceded and followed by a 1 hour rest. All these operations are made at different values of SOC (80, 60, 40 and 20\%). The equipment used for this measurement is the same battery tester previously cited. A preliminary test is necessary to determine the evolution of the $O C V$ of the battery versus its SOC. The curve is given by Figure 9. A small hysteresis of $20 \mathrm{mV}$ is observed. We decided to neglect this phenomenon in this work. An average value of this curve is used to compensate the variation of the $O C V$ during a 5 min pulse. An example of the measured and compensated signals is shown in Figure 10. A Matlab-Simulink program optimizes the diffusive impedance 


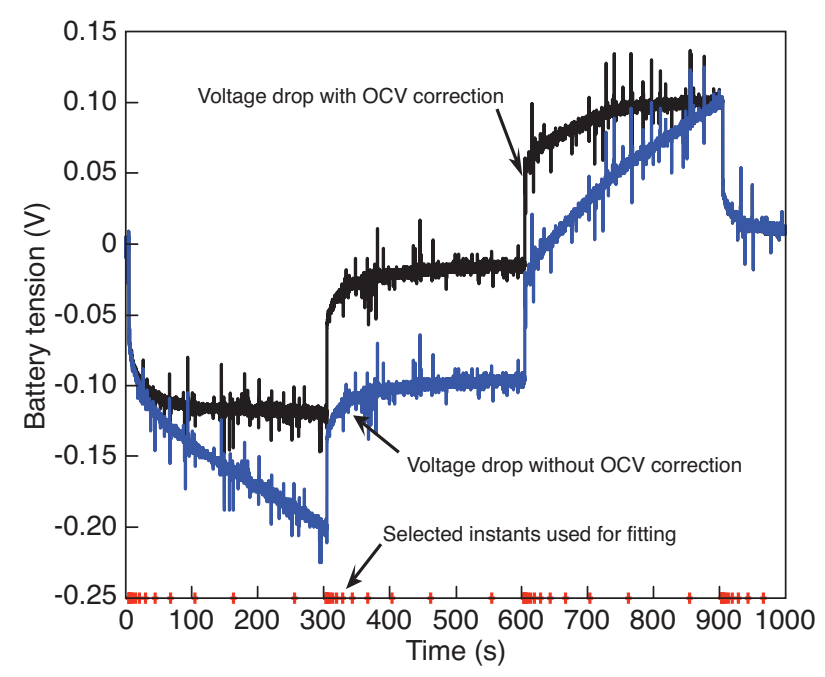

Figure 10

Voltage drop response measured on battery with and without $O C V$ correction.

parameters to fit the simulated response with the experimental data by minimising a least square criterion. In order to not limit the influence on the optimization of the long time response, the experimental data used for this optimization is selected at instants with a logarithmic distribution after each change in the current. The selected experimental data instants are also shown in Figure 10.

\subsection{Validation of the Model}

In order to validate the model of the battery, the voltage response of the model to a "validation profile" is compared to the real response of the battery. This "validation profile" is composed of a suite of charging/discharging pulses defined from experimental measurement on a real hybrid vehicle adapted to the capabilities of the tested battery.

\section{RESULTS}

\subsection{Identification of $R_{1}$ and $R_{2} / / C P E_{2}$}

As explained above, the resistance $R_{1}$ and the parameters of $R_{2} / / C P E_{2}$ are determined from impedancemetry data for different SOC. The results are represented in Figure 11 where each parameter is represented for different levels of current. The values obtained at different SOC are superposed on the same graph. We can see that $R_{1}$ is relatively constant and will be set to $1.95 \mathrm{~m} \Omega$,. Concerning the parameters of $R_{2} / / C P E_{2}$, we can say that $R_{2}$ and $Q_{2}$ depend on the current level and SOC. $R_{2}$ tends to decrease when the current increases. As $Q_{2}$ tends to have opposite variation, the time constant $\tau_{2}$ is expected to be rather constant, which is effectively observed. The limited variation of $\alpha_{2}$ can be supposed of low impact on the value of the impedance. For these reasons, we will keep $\alpha_{2}$ and $\tau_{2}$ equal to a value which will depend only on SOC. The behaviour of $R_{2}$ as a function of the current level is modelling by Equation (12). The data obtained for different SOC can be fitted with the same parameter value of $A$ and $B$. There is only $C$ which depends on SOC. The fitting are represented with experimental data for different SOC in Figure 12.

The optimized values for the parameters are $A=35.87 \mathrm{~A}$, $B=10.73 \mathrm{~V}^{-1}$. Figure 13 shows the variation of $C$ which precise values are: $C(80)=1.05 \mathrm{~m} \Omega, C(60)=1.06 \mathrm{~m} \Omega, C(40)=$ $1.18 \mathrm{~m} \Omega, C(20)=1.57 \mathrm{~m} \Omega$.

The results obtained with impedance spectroscopy at low currents and the data obtained from the high current pulses are in a good agreement (cf. Fig. 12).

\subsection{Identification of $C P E_{3}$}

Now, the parameters of the diffusive impedance $C P E_{3}\left(\omega_{1}\right.$, $\omega_{5}, \gamma$ and $p$ ) have to be evaluated from temporal data. In order to assure convergent results from optimization, we fixed the values of $\omega_{1}$ and $\omega_{5}$ :

$$
f_{1}=\frac{\omega_{1}}{2 \pi}=1 \mathrm{mHz} \text { and } f_{5}=\frac{\omega_{5}}{2 \pi}=5 \mathrm{~Hz}
$$

Moreover, for this first study, we also imposed the value $p$ of the slope at 0.5 . Consequently, only the value of $\gamma$ is adjusted depending on the value of SOC. The values of $\gamma$ and $C$ obtained at different SOC are represented in Figure 13.

The result of the optimization can be seen on the voltage chronograms represented for the different SOC in Figure 14. The good agreement is attested by a relative error about $\pm 1 \%$. As the model is identified in two parts, it is essential to verify the coherence between the results. Figure 15 shows the impedance spectra measured with the impedance analyser and the spectra of the diffusive impedance simulated after identification of $\mathrm{CPE}_{3}$.

\subsection{Validation on Current Profile}

The validation is realised at $\mathrm{SOC}=40 \%$ with $\triangle \mathrm{SOC}=0$. The measured and simulated voltages are represented in Figure 16. As in Figure 14, the quality of the result is attested by a relative error smaller than $\pm 2 \%$.

\section{DISCUSSION}

The main advantage of this work is the possibility of rapidly identifying a battery model which varies with the value of current. The use of impedance spectroscopy at "high frequencies" for different values of current is a good alternative to 

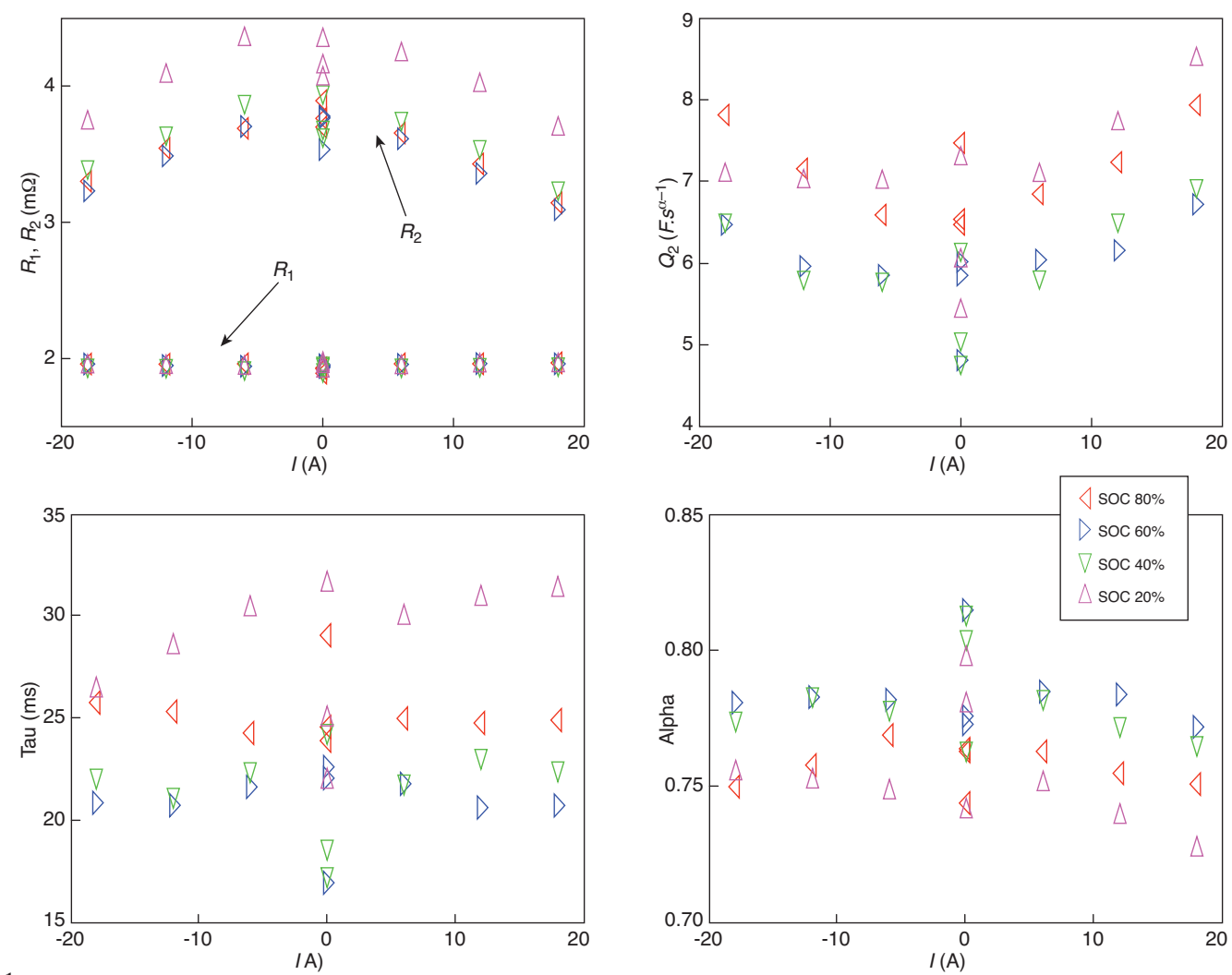

Figure 11

Values of $R_{1}, R_{2}, Q_{2}, C P E_{2}, \tau_{2}$ and $\alpha_{2}$ as function of the current level for different SOC.
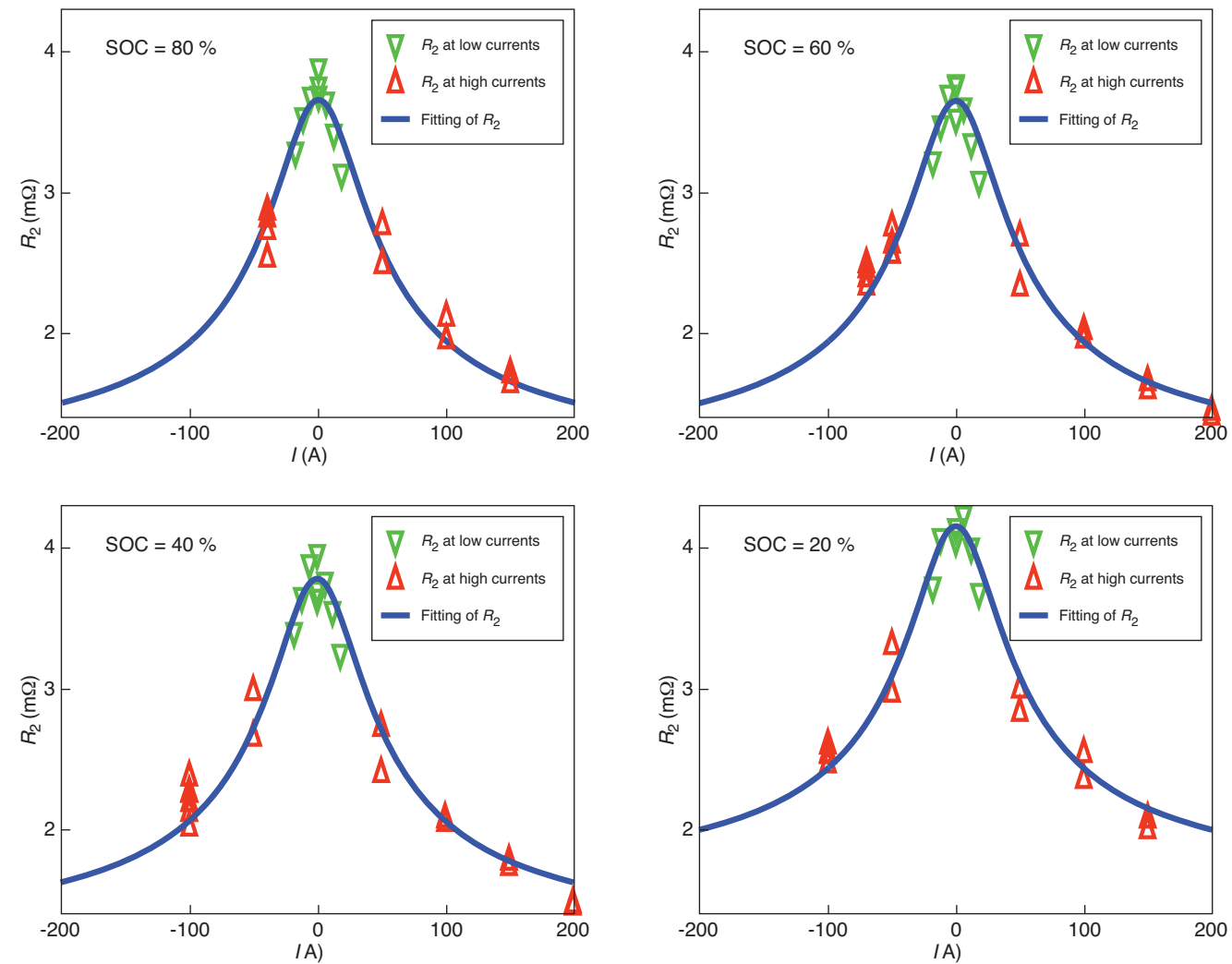

Figure 12

$R_{2}$ measured and fitted as function of the current level for different SOC. 

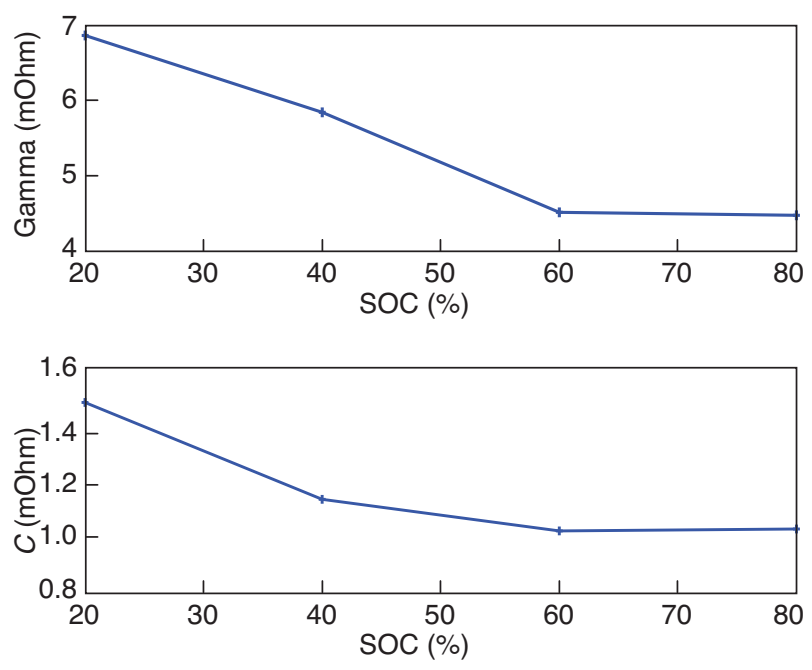

Figure 13

Variations of the values of $C$ and $\gamma$ as function of SOC.

the chronopotentiometry on pulses. Indeed, the determination of resistance from voltage profiles could be disturbed, as it was already underlined, by the rate of data acquisition, but also by the difficulty to separate the "fast" voltage response of the $R_{2} / / C P E_{2}$ from the "slow" one of the $C P E_{3}$. Even with a very fast data acquisition system, the choice of the right value for the voltage used in the resistance calculus needs to have an idea of the time constant of the $R_{2} / / C P E_{2}$. Otherwise, bad estimations are expected.

In this work, we had opportunity to check that the voltage measured on the front of a pulse after a time equals to $5 \tau_{2}$ gives values for the resistance in agreement with the values obtained by spectroscopy ( $c f$. Fig. 17). By this way, the voltage variation caused by $R_{2} / / C P E_{2}$ is finished and the one caused by $\mathrm{CPE}_{3}$ is minimum. In the present method only one or two measurements on high current pulses are required to complete the medium current measurements by spectroscopy. If one has a powerful analyser (with a $100 \mathrm{~A}$ amplifier for instance), the spectroscopic measurements could be sufficient to completely identify $R_{2}(i)$.

Some eventual improvements of the method must be studied. The present work does not take into account the experimental uncertainties in measurements and their influence on the final result. The hysteresis had been neglected. The results ( $c f$. Fig. 9) show a $20 \mathrm{mV}$ gap in $O C V$ curve (for a voltage element of $3.7 \mathrm{~V}$ ) which is far less than hysteresis in NiMH battery for instance (about $60 \mathrm{mV}$ for a $1.3 \mathrm{~V}$ element). This result is deduced from measurements of the voltage at different values of SOC. Each measurement occurs after one hour of rest which is
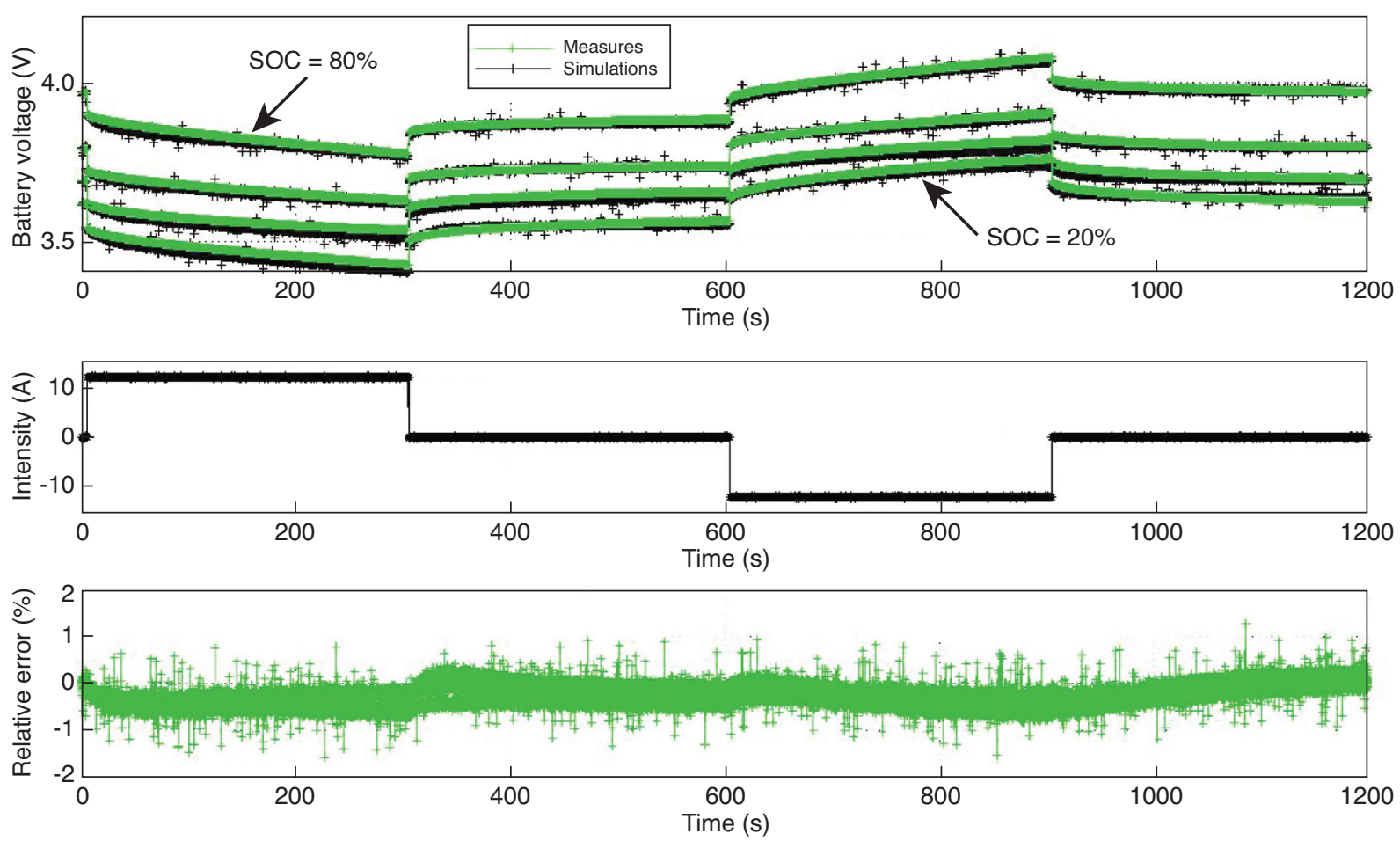

Figure 14

Simulated and measured voltages after the optimisation on the $5 \mathrm{~min}$ pulse at $1 C$ for different SOC. 

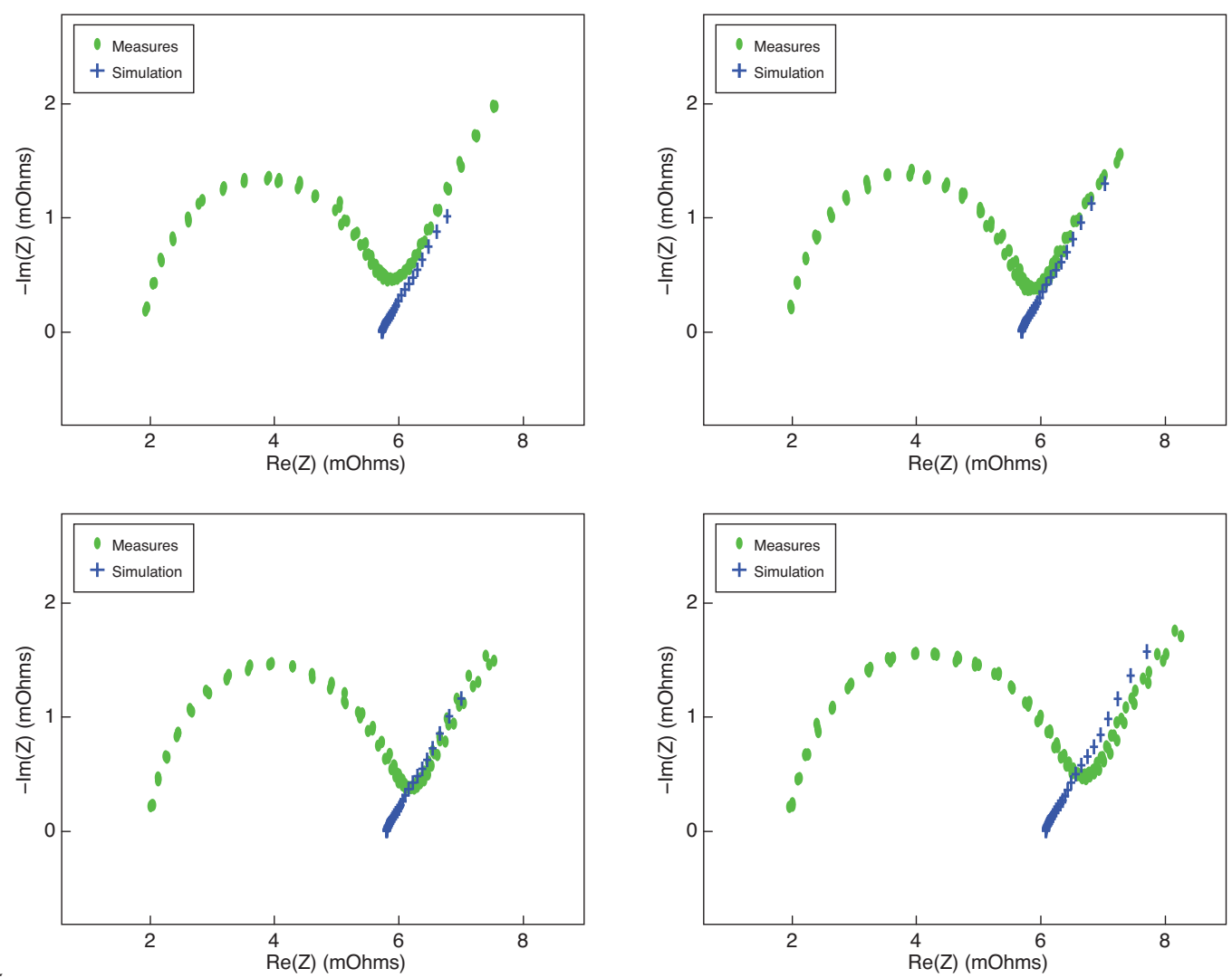

Figure 15

Simulated and measured impedance spectra after the optimisation on the 5 min pulse at $1 C$.
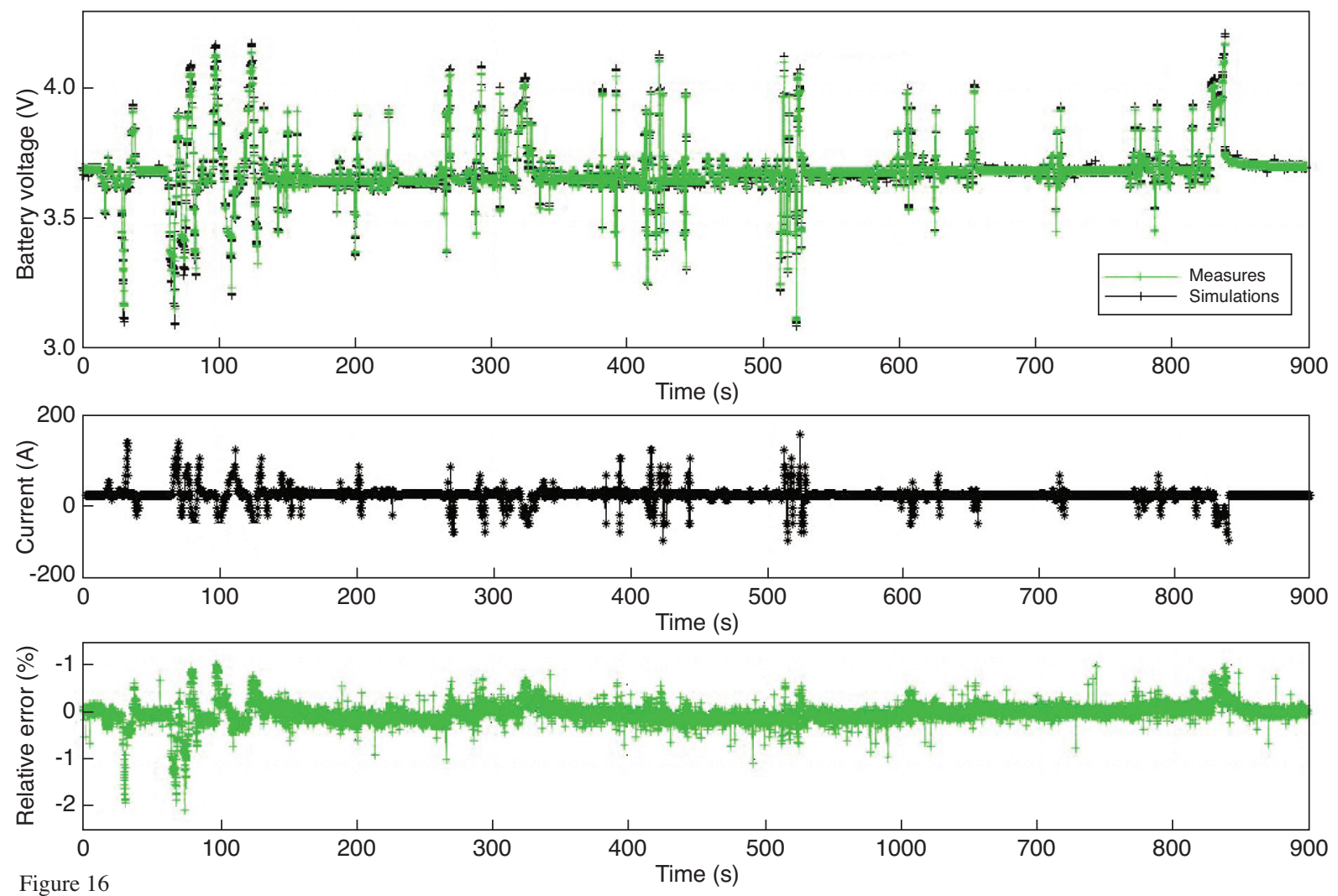

Validation of the battery model on a profile of current. 

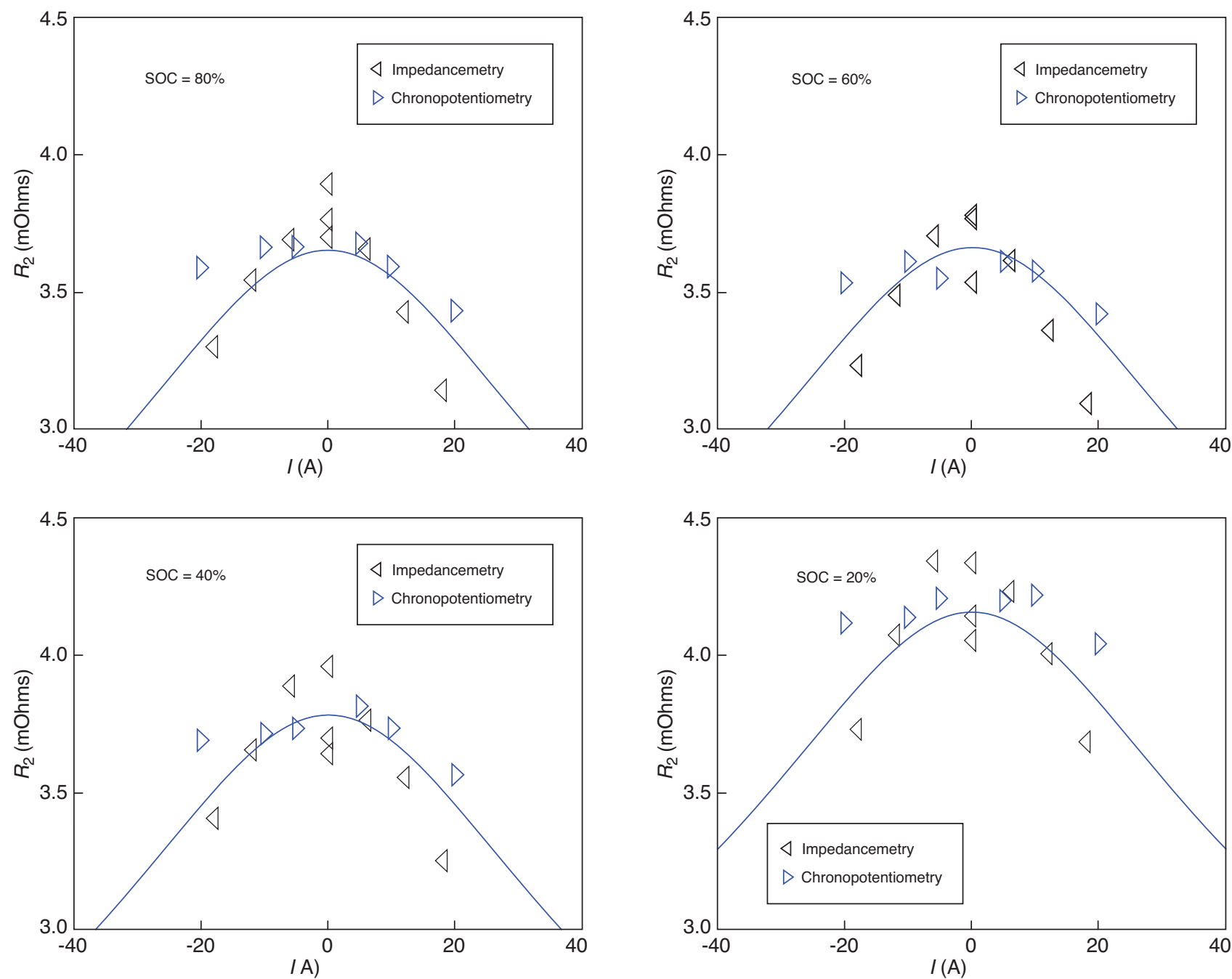

Figure 17

Superposition of $R_{2}$ measured by impedancemetry and $R_{2}$ measured by chronopotentiometry.

supposed to be long enough to reach thermodynamic equilibrium. New experiments with longer rest time will enable to verify if this apparent hysteresis is a real one.

Furthermore, the influence of the temperature will be a part of a future work.

The good agreement between measured and simulated voltage in Figure 16 tends to prove the validity of the method, but it would be a good idea to go deeper into the question of coherence between the spectrum measured and the one simulated ( $c f$. Fig. 15). Are the differences due to inappropriate measurement conditions (low data acquisition rate during the pulse for example) or to physical reasons (like influence of the level of current on the diffusive impedance)?

\section{ACKNOWLEDGMENTS}

The authors want to thank ADEME (Agence pour le Développement et la Maîtrise de l'Énergie) which financed a $\mathrm{PhD}$ grant. Thanks are also due to J.P. Diard from INPG (Institut National Polytechnique de Grenoble) and F. Badin from IFP (Institut français du pétrole) who gave some useful advice. We also want to thank J. Scordia from LMS-Imagine for his help. Discussions with other partners of the program SIMSTOCK were helpful too.

\section{REFERENCES}

1 Buller S., Thele M., Karden E., De Doncker R.W. (2003) Impedance-based non-linear dynamic battery modelling for automotive applications, J. Power Sources 113, 422-430. 
2 Freedom CAR Battery Test Manual for Power-Assist Hybrid Electric Vehicles, DOE/ID-11069, October 2003.

3 Johnson V.H. (2002) Battery performance models in ADVISOR, J. Power Sources 110, 2, 321-329.

4 Sabatier J., Aoun M., Oustaloup A., Grégoire G., Ragot F., Roy P. (2006) Fractional system identification for lead acid battery state of charge estimation, Signal Process. 86, 2645-2657.

5 Plett G.L. (2004) Extended Kalman filtering for battery management systems of LiPB-based HEV battery packs, Part 2. Modeling and identification, J. Power Sources 134, 262-276.

6 Barsoukov E., Macdonald J.R. (2005) Impedance Spectroscopy, Second Edition, Wiley-Interscience.

7 Barsoukov E., Kim J.H., Kim D.H., Hwang K.S., Yoon C.O., Lee H. (2000) Parametric analysis using impedance spectroscopy: relationship between material properties and battery performance, J. New Mat. Electr. Sys. 3, 301-308.

8 Itagaki M., Kobari N., Yotsuda S., Watanabe K., Kinoshita S., Ue M. (2004) In situ electrochemical impedance spectroscopy to investigate negative electrode of lithium-ion rechargeable batteries, J. Power Sources 135, 255-261.

9 Bundy K., Karlsson M., Lindbergh G., Lundqvist A. (1998) An electrochemical impedance spectroscopy method for prediction of the state of charge of a nickel-metal hydride battery at open circuit and during discharge, J. Power Sources 72, 118-125.

10 Blanke H., Bohlen O., Buller S., De Doncker R.W., Fricke B., Hammouche A., Linzen D., Thele M., Sauer D.U. (2005) Impedance measurements on lead-acid batteries for state-ofcharge, state-of health and cranking capability prognosis in electric and hybrid electric vehicles, J. Power Sources 144, 2, 418-425.
11 Kuhn E., Forgez C., Friedrich G., Macret P. (2005) Comparison between two NiMH battery models. EVS 21, Monaco, April 2005.

12 Karden E., Buller S., De Doncker R.W. (2002) A frequencydomain approach to dynamical modelling of electrochemical power sources, Electrochim. Acta 47, 2347-2356.

13 Thele M., Bohlen O., Sauer D.U., Karden E. (2008) Development of a voltage-behaviour model for NiMH batteries using an impedance based modelling concept, J. Power Sources 175, 635-643.

14 Mauracher P., Karden E. (1997) Dynamic modelling of lead/acid batteries using impedance spectroscopy for parameter identification, J. Power Sources 67, 69-84.

15 Takano K., Nozaki K., Saito Y., Negshi A., Kato K., Yamagushi Y. (2000) Simulation study of electrical dynamic characteristics of lithiumion battery, J. Power Sources 90, 214-223.

16 Tenno A., Tenno R., Suntio T. (2004) A Method for Battery Impedance Analysis, J. Electrochem. Soc. 151, 6, A806- A824.

17 Kuhn E., Forgez C., Lagonotte P., Friedrich G., (2006) Modelling Ni-MH battery using Cauer and Foster structures, J. Power Sources 158, 2, 1490-1497.

18 Oustaloup A., Cois O., Le Lay L. (2005) Représentation et identification par modèle non entier, Lavoisier, Paris.

19 Diard J.P., Le Gorrec B., Montella C. (1998) EIS study of electrochemical battery discharge on constant load, J. Power Sources 70,78-84.

Final manuscript received in May 2009 Published online in November 2009 